\title{
Spin-orbit interaction determined by antilocalization in an InSb quantum well
}

\author{
R. L. Kallaher* and J. J. Heremans ${ }^{\dagger}$ \\ Department of Physics, Virginia Tech, Blacksburg, Virginia 24061, USA
}

N. Goel, S. J. Chung, and M. B. Santos

Homer L. Dodge Department of Physics and Astronomy, University of Oklahoma, Norman, Oklahoma 73019, USA

(Received 10 July 2009; revised manuscript received 21 September 2009; published 2 February 2010)

\begin{abstract}
The magnetoresistance at temperatures below $20 \mathrm{~K}$ in an $n$ - InSb/ $\operatorname{In}_{0.85} \mathrm{Al}_{0.15} \mathrm{Sb}$ two-dimensional electron system is studied and described in terms of antilocalization due to quantum interference under strong spin-orbit interaction. The spin-orbit interaction coefficients are extracted by fitting the magnetoresistance data to an antilocalization theory distinguishing the Rashba and Dresselhaus contributions. A good agreement between magnetoresistance data and theory suggests a Rashba coefficient $|\alpha| \approx 0.03 \mathrm{eV} \AA$ and a Dresselhaus coefficient $\gamma \approx 490 \mathrm{eV} \AA^{3}$. A strong contribution from the Dresselhaus term leads to pronounced anisotropy in the energy splitting induced by spin-orbit interaction in the two-dimensional electron dispersion.
\end{abstract}

DOI: 10.1103/PhysRevB.81.075303

PACS number(s): 73.61.Ey, 73.20.Fz, 72.25.Rb

\section{INTRODUCTION}

In the study of materials where spin-dependent phenomena are prevalent, InSb stands out due to characteristics such as a strong spin-orbit interaction (SOI), a high effective $g$ factor, and a high electron mobility. InSb and InSb quantum wells (QWs) have drawn attention in several developments, ranging from the quest for new electronic devices, ${ }^{1,2}$ to magnetoresistive devices ${ }^{3,4}$ and spin-based electronics. ${ }^{5}$ Here we present low-temperature measurements of the spindependent quantum-interference effect of antilocalization $(\mathrm{AL})$ in InSb quantum wells. The measurements allow a comparison with theory and a determination of the SOI parameters.

Quantum interference between partial electron waves on exact time-reversed trajectories leads to the phenomena of AL (Refs. 6-11) and weak localization (WL) (Refs. 6, 7, and 12) in low-temperature electronic transport. WL is characteristic of constructive interference of wave functions at low applied magnetic fields $H$, and $\mathrm{AL}$ of destructive interference. ${ }^{6-12}$ The interference is governed by SOI and the phase coherence time $\tau_{\varphi}$, which determines the time scale available for interference. AL, in particular, can be pictured as resulting from destructive interference of spin-1/2 carriers locked onto a closed path by SOI. ${ }^{7}$ AL magnetotransport measurements thus form a valuable experimental tool to access SOI parameters and $\tau_{\varphi}$ in semiconductor systems. ${ }^{9-11,13-27}$ In this context, we present low-field magnetotransport measurements on an InSb two-dimensional electron system (2DES) in an $\mathrm{InSb} / \mathrm{In}_{0.85} \mathrm{Al}_{0.15} \mathrm{Sb}$ heterostructure at temperatures $T<20 \mathrm{~K}$. The observed dependence of the conductivity on $H$ is discussed in terms of AL, and enables us to determine the dominant SOI terms, and to compare our findings with the literature on $n$-type InSb quantum wells.

SOI in (001)-grown III-V 2DESs is described by the Hamiltonian $\mathcal{H}_{\mathcal{S O}}=\hbar \boldsymbol{\sigma} \cdot \boldsymbol{\Omega}(\boldsymbol{k}),{ }^{10,19,25-32}$ where $\boldsymbol{\sigma}$ $=\left(\sigma_{x}, \sigma_{y}, \sigma_{z}\right)$ is the vector of Pauli spin matrices and $\boldsymbol{k}$ represents the wave vector. Following Ref. 10, we introduce the wave vector in the $2 \mathrm{DES}$ plane, $k_{p}=\sqrt{k_{x}^{2}+k_{y}^{2}}$ and $\theta$ $=\tan ^{-1}\left(k_{x} / k_{y}\right)$, where $\theta=0$ corresponds to $\boldsymbol{k} \|[100] . \boldsymbol{\Omega}(\boldsymbol{k})$ is composed of a sum,

$$
\boldsymbol{\Omega}(\boldsymbol{k})=\boldsymbol{\Omega}^{R}+\boldsymbol{\Omega}_{\theta}^{D}+\boldsymbol{\Omega}_{3 \theta}^{D},
$$

where ${ }^{10,19,25-27,29-32}$

$$
\begin{gathered}
\hbar \boldsymbol{\Omega}^{R}=\alpha k_{p}\left(\begin{array}{c}
\sin \theta \\
-\cos \theta
\end{array}\right), \\
\hbar \boldsymbol{\Omega}_{\theta}^{D}=\gamma k_{p}\left(\left\langle k_{z}^{2}\right\rangle-\frac{k_{p}^{2}}{4}\right)\left(\begin{array}{c}
-\cos \theta \\
\sin \theta
\end{array}\right), \\
\hbar \boldsymbol{\Omega}_{3 \theta}^{D}=-\gamma \frac{k_{p}^{3}}{4}\left(\begin{array}{c}
\cos 3 \theta \\
\sin 3 \theta
\end{array}\right) .
\end{gathered}
$$

Here $\alpha$ represents the Rashba coefficient, $\gamma$ the Dresselhaus coefficient, and $\left\langle k_{z}^{2}\right\rangle$ the square of the quantized wave vector in the direction perpendicular to the 2DES plane. The Rashba term, $\boldsymbol{\Omega}^{R}$, arises from the structural asymmetry of the heterostructure. ${ }^{10,19,25-32}$ The coefficient $\alpha$ varies with the average electric field across the well and with contributions from the interfaces between quantum well and barrier layers. ${ }^{10,25-33}$ Theoretical studies of asymmetric $n$-InSb/InAlSb quantum wells indicate that interface contributions to $\alpha$ dominate. ${ }^{28}$ The Dresselhaus term $\left(\boldsymbol{\Omega}^{D}=\boldsymbol{\Omega}_{\theta}^{D}\right.$ $\left.+\boldsymbol{\Omega}_{3 \theta}^{D}\right)$ arises from the lack of inversion symmetry in the zinc-blende crystal structure. ${ }^{34}$ Compared to other III-V semiconductors, bulk InSb has a high value for $\gamma_{b u l k}$, theoretically predicted to be in the range $560 \lesssim \gamma_{\text {bulk }}$ $\lesssim 760 \mathrm{eV} \AA$. $^{28,35,36}$ Therefore, $\boldsymbol{\Omega}^{D}$ may show a substantial impact on $\mathrm{AL}$ in InSb-based systems, and in turn AL can provide experimental insight in $\boldsymbol{\Omega}^{D}$ and provide values for $\gamma$.

\section{EXPERIMENT}

The InSb 2DES studied in this work, schematically depicted in Fig. 1, was grown by molecular-beam epitaxy on semi-insulating GaAs (001) substrate. From the substrate upward, the heterostructure consists of a 215-nm-thick AlSb 


\begin{tabular}{|c|}
\hline InSb $(13 \mathrm{~nm})$ \\
\hline$-\delta$-doped $\mathrm{Si}$ \\
\hline $\mathbf{I n}_{\mathbf{0 . 8 5}} \mathbf{A l}_{\mathbf{0 . 1 5}} \mathbf{S b}(\mathbf{1 5 0} \mathbf{~ n m})$ \\
\hline Ims l l \\
\hline$\delta$-doped $\mathrm{Si}$ \\
\hline $\mathrm{In}_{0.85} \mathrm{Al}_{0.15} \mathrm{Sb}(4.1 \mu \mathrm{m})$ \\
\hline AlSb $(215 \mathrm{~nm})$ \\
\hline GaAs (001) \\
\hline
\end{tabular}

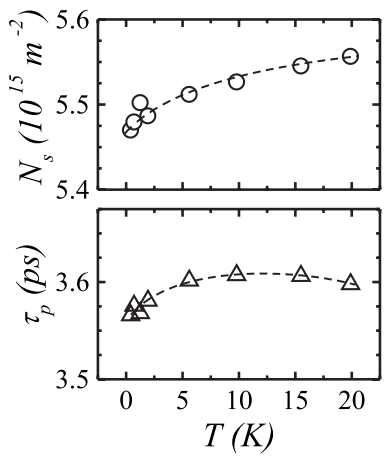

FIG. 1. Left: Schematic of the InSb/InAlSb 2DES heterostructure. The $\delta$-doped layers are located at the following positions: 40 $\mathrm{nm}$ below, $40 \mathrm{~nm}$ above, and $140 \mathrm{~nm}$ above the quantum well (QW). Right: Measured carrier concentration $N_{s}$ and momentum scattering time $\tau_{p}$ as a function of $T$. Dashed lines are guides to the eyes.

buffer, a $4.1-\mu \mathrm{m}$-thick $\operatorname{In}_{0.85} \mathrm{Al}_{0.15} \mathrm{Sb}$ layer, the 25 -nm-thick InSb quantum well, a $150 \mathrm{~nm} \operatorname{In}_{0.85} \mathrm{Al}_{0.15} \mathrm{Sb}$ layer, and a 13 $\mathrm{nm}$ undoped $\mathrm{InSb}$ cap layer. Electrons are provided to the well by two Si $\delta$-doped layers, at $40 \mathrm{~nm}$ below and $40 \mathrm{~nm}$ above the well. A third $\delta$-doped layer, $23 \mathrm{~nm}$ below the surface, passivates surface states. The strength of the Rashba term [Eq. (1a)], defined by $\alpha$, depends on band parameters $^{35,37}$ and on several sources of asymmetry in the heterostructure, and can vary substantially between heterostructures. In the present structure, Fermi-level pinning at the surface as well as the presence of two $\delta$-doped layers above the quantum well contribute to asymmetry, and contribute to a sizable $\alpha$ via the large SOI in InSb. Spin-dependent mesoscopic transport experiments allowed an approximate value $\alpha \approx 0.1 \mathrm{eV} \AA$ to be deduced in similar heterostructures. ${ }^{5}$ Measurements on $n$-InSb/InAlSb 2DESs which are remotely doped from only one side of the well have determined 0.07 $\lesssim|\alpha| \lesssim 0.14 \mathrm{eV} \AA{ }^{38,39}$ In the sections below the AL data will be analyzed to obtain the magnitude of $\alpha$ in the heterostructure shown in Fig. 1.

Magnetotransport was measured using standard lowfrequency lock-in techniques between 0.4 and $20 \mathrm{~K}$. The data and analysis presented here were obtained on a 100 $\times 20 \mu \mathrm{m}$ Hall bar fabricated by photolithography and wet etching. Other samples fabricated from the same wafer yield similar results. Resistivity and Hall-effect measurements indicate that the electron areal density $N_{s} \approx 5.5 \times 10^{15} \mathrm{~m}^{-2}$ and the electron mobility $\mu \approx 8.9 \mathrm{~m}^{2} / \mathrm{V} \mathrm{s}$ at $0.4 \mathrm{~K}$. Hall-effect measurements do not show evidence of two-band transport and single-subband occupancy is thus assumed. The parameter $\left\langle k_{z}^{2}\right\rangle$ for the first quantized level can be obtained from finite square-well solutions ${ }^{40}$ while accounting for nonparabolicity in the InSb conduction band and using the respective band-edge offsets of the well and barrier materials. ${ }^{28,41,42}$ The calculation yields $\left\langle k_{z}^{2}\right\rangle=8 \times 10^{15} \mathrm{~m}^{-2}$, the value used in the remainder of this work. Allowing for nonparabolicity, the effective mass $m^{*},\left\langle k_{z}^{2}\right\rangle, N_{s}$, and $\mu$ are used to calculate other transport parameters, such as the mobility scattering time $\tau_{p}$ and the two-dimensional diffusion constant $D$. From $\left\langle k_{z}^{2}\right\rangle$ and $N_{s}$ we can estimate the strength of $\boldsymbol{\Omega}^{D}$ at the Fermi energy

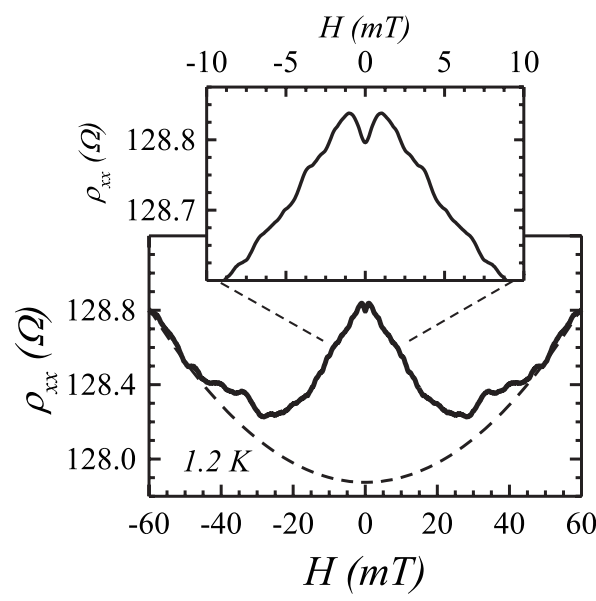

FIG. 2. Low-field changes in resistivity at $T=1.2 \mathrm{~K}$. The dashed line indicates the parabolic background. The inset shows positive magnetoresistance around $H=0$ characteristic of AL.

$E_{F}$, using $k_{p}^{2}=k_{F}^{2}=2 \pi N_{s}$, with $k_{F}$ the Fermi wave vector. For the present heterostructure, the values for $\left\langle k_{z}^{2}\right\rangle$ and $N_{s}$ lead to $\left|\left[\left\langle k_{z}^{2}\right\rangle-\left(\pi N_{s} / 2\right)\right]\right| \ll\left|\left(\pi N_{s} / 2\right)\right|$, and hence from Eqs. (1b) and (1c) we conclude that $\left|\boldsymbol{\Omega}_{\theta}^{D}\right| \ll\left|\boldsymbol{\Omega}_{3 \theta}^{D}\right|$. Since the term $\boldsymbol{\Omega}_{3 \theta}^{D}$ dominates, the term $\boldsymbol{\Omega}_{\theta}^{D}$ will be neglected in the AL analysis.

Figure 2 contains an example of the low-field magnetoresistance obtained at $T=1.2 \mathrm{~K}$. In order to account for slight Hall-effect contributions to the data due to inevitable contact misalignment, the component antisymmetric in $H$ has been subtracted from the data. For $0 \leqq H \lesssim 30 \mathrm{mT}$ the sample exhibits the positive magnetoresistance transitioning to negative magnetoresistance characteristic of AL. At higher $H(H \geqslant 30 \mathrm{mT})$ an $\sim H^{2}$ dependence of the magnetoresistance is observed, typical of geometric magnetoresistance in high- $\mu$ materials, ${ }^{3}$ and is accounted for in order to ensure accuracy of the AL analysis.

AL (Refs. 6, 8-11, 19, 25, 26, and 43) is expressed as a change in longitudinal conductivity, $\sigma_{x x}$. Ignoring electronelectron interactions, the $H$ dependence of $\sigma_{x x}$ is contained in the sum of a classical term and the AL correction term, ${ }^{12}$

$$
\sigma_{x x}(H)=\frac{\sigma_{0}}{1+(\mu H)^{2}}+\Delta \sigma(H)
$$

where $\sigma_{0}=N_{s} e^{2} \tau_{p} / m^{*}$ denotes the zero- $H$ Drude conductivity and $\Delta \sigma(H)$ the AL correction. The experiment measures the longitudinal resistivity $\rho_{x x}(H)$, and the following steps are followed to determine $\Delta \sigma(H)$ from $\rho_{x x}(H)$. First a purely quadratic term $\sim H^{2}$ is fit to the high-field data (Fig. 2), and the $H^{2}$ term is subtracted from the measured $\rho_{x x}$ to determine $\Delta \rho(H) . \Delta \rho(H)$ denotes the AL correction to $\rho_{x x}$. Then $\Delta \sigma(H)$ is determined using $\Delta \sigma(H) \approx-\Delta \rho(H) \rho_{0}^{-2}$, where $\rho_{0} \equiv \sigma_{0}^{-1}$.

Examples of $\Delta \sigma(H)$ are displayed in Fig. 3. The data exhibits the characteristic shape of AL, with an initial decrease in conductivity from $H=0$, reaching a minimum at $H=H_{\text {min }} \approx 1.1 \mathrm{mT}$, after which positive magnetoconductivity is observed. The $\Delta \sigma(H)$ curves are parametrized in terms of $\tau_{p}$, the phase coherence time $\tau_{\varphi}$, and in terms of the various contributions to $\boldsymbol{\Omega}(\boldsymbol{k})$. Specifically, $\Delta \sigma(H)$ was fit to an 


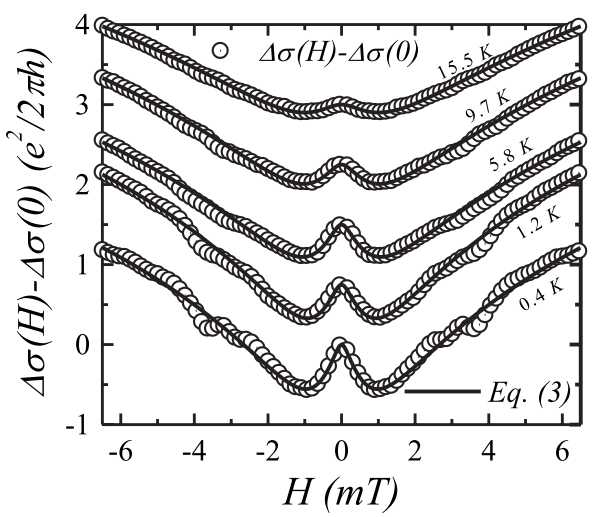

FIG. 3. Changes in conductivity derived from the data at different $T$, along with the fits obtained from Eq. (3). The curves have been offset for clarity.

AL expression which separately accounts for $\boldsymbol{\Omega}^{R}$ and $\boldsymbol{\Omega}_{3 \theta}^{D},{ }^{10,26}$ and which allows us to examine their respective magnitudes and dependence on $T$.

\section{ANALYSIS}

In 2DESs where $\boldsymbol{\Omega}_{\theta}^{D}$ can be neglected, the AL correction to the conductivity in perpendicular $H$ can be expressed as $^{10,26}$

$$
\begin{aligned}
\Delta \sigma(H)= & \frac{-e^{2}}{4 \pi^{2} \hbar}\left[3 C+\frac{1}{a_{0}}+\frac{2 a_{0}+1+b_{s}}{a_{1}\left(a_{0}+b_{s}\right)-2 b_{s}^{\prime}}-\sum_{n=1}^{\infty} \frac{3}{n}\right. \\
& -\frac{3 a_{n}^{2}+2 b_{s} a_{n}-1-2(2 n+1) b_{s}^{\prime}}{\left(a_{n}+b_{s}\right) a_{n-1} a_{n+1}-2 b_{s}^{\prime}\left[(2 n+1) a_{n}-1\right]} \\
& \left.+2 \ln \left(b_{t r}\right)+\Psi\left(\frac{1}{2}+b_{\varphi}\right)\right]
\end{aligned}
$$

where $\Psi$ represents the digamma function; $C$ is Euler's constant; $\quad a_{n}=b_{\varphi}+b_{s}+n+(1 / 2) ; \quad b_{t r}=\left(\hbar / 4 e D \tau_{p} H\right) ; \quad b_{s}^{\prime}$ $=\left(2 \hbar\left|\mathbf{\Omega}^{R}\right|^{2} \tau_{1} / 4 e D H\right) ; b_{s}=b_{s}^{\prime}+\left(2 \hbar\left|\boldsymbol{\Omega}_{(3 \theta)}^{D}\right|^{2} \tau_{3}\right) / 4 e D H ;$ and $b_{\varphi}$ $=\left(\hbar / 4 e D \tau_{\varphi} H\right)$. The relaxation times $\tau_{n}(n=1,3)$ are expressed as ${ }^{10,30,44}$

$$
\frac{1}{\tau_{n}}=\int W(\zeta)[1-\cos (n \zeta)] d \zeta,
$$

where $W(\zeta)$ denotes the scattering probability, dependent on the scattering angle $\zeta$. We note that for degenerate systems $\tau_{1}=\tau_{p} .{ }^{31}$ The value of $\left(\tau_{1} / \tau_{3}\right)=\left(\tau_{p} / \tau_{3}\right)$ in 2DESs ranges from $\left(\tau_{1} / \tau_{3}\right)=1$ for isotropic scattering to $\left(\tau_{1} / \tau_{3}\right)=9$ for smallangle scattering. ${ }^{10,30,44}$ Although Eq. (3) is derived for the diffusive regime $\left(b_{t r}>1\right)$, previous AL experiments demonstrate that nominally diffusive formalisms accurately describe SOI in high- $\mu$ 2DES. ${ }^{15}$ Equally satisfactory fits to our experimental data were, in fact, not obtained with theories aiming beyond the diffusive regime. ${ }^{9,11}$

With $b_{t r}$ determined from $N_{s}$ and $\rho_{0}$, the experimental $\Delta \sigma(H)-\Delta \sigma(0)$ for $|H|<6.5 \mathrm{mT}$ at different $T$ were fit to Eq. (3), allowing us to separately assess $\boldsymbol{\Omega}^{R}$ and $\boldsymbol{\Omega}_{3 \theta}^{D}$ (neglecting $\boldsymbol{\Omega}_{\theta}^{D}$ ). Fits to Eq. (3) were performed while varying $b_{s}, b_{s}^{\prime}$, and

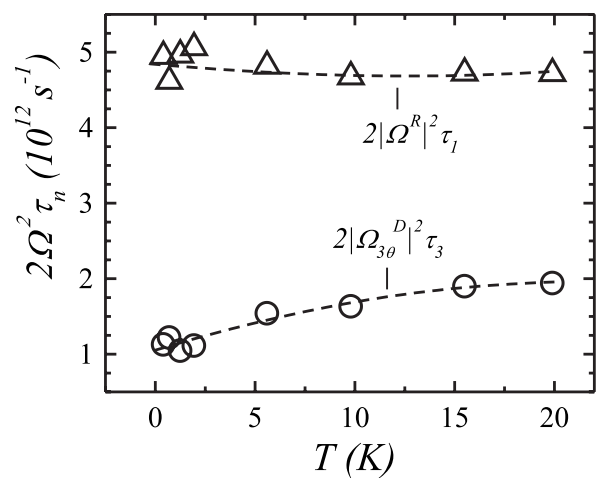

FIG. 4. The terms $2 \Omega^{2} \tau_{n}$ extracted from fits of $\Delta \sigma(H)-\Delta \sigma(0)$ to Eq. (3). From the $2 \Omega^{2} \tau_{n}$ corresponding contributions to $\boldsymbol{\Omega}$ can be deduced. Dashed lines form guides to the eyes.

$b_{\varphi}$, and the total error in the values thus obtained are estimated at $\sim 10 \%$. From $b_{s}$ and $b_{s}^{\prime}$ we can deduce $2\left|\Omega^{R}\right|^{2} \tau_{1}$ and $2\left|\boldsymbol{\Omega}_{3 \theta}^{D}\right|^{2} \tau_{3}$, and hence, with knowledge of $\tau_{1}=\tau_{p}$ and $\tau_{3}$, we obtain $\left|\boldsymbol{\Omega}^{R}\right|$ and $\left|\boldsymbol{\Omega}_{3 \theta}^{D}\right|$. The results are presented below.

\section{RESULTS AND DISCUSSION}

Examples of the fits of Eq. (3) to the experimental $\Delta \sigma(H)-\Delta \sigma(0)$ are displayed along with the corresponding data in Fig. 3. Equation (3) accurately models $\Delta \sigma(H)$ $-\Delta \sigma(0)$ at all $T$, with a slight deviation at an oscillatory structure occurring at $H \approx \pm 3 \mathrm{mT}$ in our data. The strong $T$ dependence of the oscillatory structure, which becomes quite weak above $\approx 5 \mathrm{~K}$, suggests a phase coherence phenomenon is responsible. Although the exact origin of the observed oscillatory structure forms the subject of future study, here we offer one possible explanation. One expects universal conduction fluctuations to be averaged out due to the size of the device. However, the heterostructure shows a low density of pyramidal structural defects (associated with threading dislocations) which are not expected to affect mobility due to their low density and extended mesoscopic size. ${ }^{45}$ A hypothetical origin for the oscillatory magnetoresistance lies in Altshuler-Aronov-Spivak oscillations ${ }^{46}$ occurring for electron trajectories encompassing such mesoscopic defects, as also observed in lithographic antidot lattices. ${ }^{47}$ In the present case the irregular defect size and spacing preclude welldeveloped oscillations while still allowing for magnetoresistance effects associated with orbits of defined enclosed magnetic flux. AL phenomena do not encompass such oscillatory structure, and hence the structure is ignored for the remainder of the present work.

Figure 4 depicts the terms in Eq. (3) originating from different SOI contributions, $2\left|\boldsymbol{\Omega}^{R}\right|^{2} \tau_{1}$ and $2\left|\boldsymbol{\Omega}_{3 \theta}^{D}\right|^{2} \tau_{3}$ as obtained from the fits to the data. The magnitude of $2\left|\mathbf{\Omega}^{R}\right|^{2} \tau_{1}$ $=4 \pi N_{s} \alpha^{2} \hbar^{-2} \tau_{p}$ exceeds $2\left|\Omega_{3 \theta}^{D}\right|^{2} \tau_{3}=\left(\pi N_{s}\right)^{3} \gamma^{2} \hbar^{-2} \tau_{3}$, indicating that Rashba SOI provides the largest contribution to $\Delta \sigma(H)-\Delta \sigma(0)$. However, accounting for the contribution from $\boldsymbol{\Omega}_{3 \theta}^{D}$ is necessary for correct modeling of $\Delta \sigma(H)$ $-\Delta \sigma(0)$, with increased relevance at higher $T$. Moreover, as indicated below, both $\boldsymbol{\Omega}_{3 \theta}^{D}$ and $\boldsymbol{\Omega}^{R}$ play important roles in the energy dispersion of the $2 \mathrm{DES}$. We extract $|\alpha| \approx 0.03 \mathrm{eV} \AA$ 


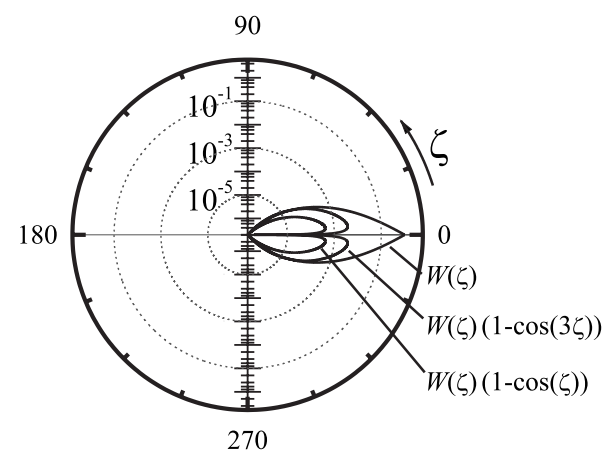

FIG. 5. Calculated angular dependence of $W(\zeta), W(\zeta)[1$ $-\cos (\zeta)]$, and $W(\zeta)[1-\cos (3 \zeta)]$ normalized to $W(0)$, from Eq. (5). The radial axis follows a logarithmic scale.

from the observed value of $2\left|\mathbf{\Omega}^{R}\right|^{2} \tau_{1} \approx 4.8 \times 10^{12} \mathrm{~s}^{-1}$. This value for $|\alpha|$ lies within the expected range, and bears comparison with recent theoretical calculations predicting $\left|\alpha^{\text {th }}\right|$ $\approx 0.04 \mathrm{eV} \AA$ for $n$-InSb/InAlSb 2DESs with equal barrier heights on both sides of the well. ${ }^{28}$

Interpretation of $2\left|\boldsymbol{\Omega}_{3 \theta}^{D}\right|^{2} \tau_{3}$ requires a discussion of $\left(\tau_{1} / \tau_{3}\right)$. The ratio $\left(\tau_{1} / \tau_{3}\right)$ depends on the scattering mechanism. ${ }^{10,30,44}$ In accordance with previous studies of electronic transport in 30-nm-wide $n$-InSb/InAlSb 2DESs with high $\mu,{ }^{48}$ remote-ionized impurity scattering from $\mathrm{Si}$ ions in the $\delta$-doped layers is expected to dominate electron scattering at low $T$. For 2DESs under remote-ionized impurity scattering, the angular dependence of $W(\zeta)$ can be approximated by ${ }^{40,48}$

$$
W(\zeta) \propto \frac{\exp (-2 q d)}{\left(q+q_{T F}\right)^{2}},
$$

where the scattering wave vector $q=\left|2 k_{F} \sin (\zeta / 2)\right|$, and $d$ $=40 \mathrm{~nm}$ denotes the distance separating the 2DES from the ionized impurities. With an effective Bohr radius $a_{B}$ $=67 \mathrm{~nm}$, the Thomas-Fermi screening wave vector $q_{T F}$ $=\left(2 / a_{B}\right)=2.9 \times 10^{7} \mathrm{~m}^{-1}$. Figure 5 contains the angular dependences of $W(\zeta), W(\zeta)[1-\cos (\zeta)]$, and $W(\zeta)[1-\cos (3 \zeta)]$ normalized to $W(0)$, in a polar graph. $W(\zeta)$ is strongly peaked toward small $\zeta$, as expected for the predominantly small-angle scattering from remote-ionized impurities. As compared to $[1-\cos (\zeta)]$, the multiplicative effect of the factor $[1-\cos (3 \zeta)]$ is strongest at small $\zeta$, leading to a higher ratio $\left(\tau_{1} / \tau_{3}\right)$ for small-angle scattering than for large-angle scattering.

From Eqs. (4) and (5) we calculate $\left(\tau_{1} / \tau_{3}\right) \approx 9$. From the observed $2\left|\boldsymbol{\Omega}_{3 \theta}^{D}\right|^{2} \tau_{3} \approx 1.1 \times 10^{12} \mathrm{~s}^{-1}$ at $0.4 \mathrm{~K}$ we then extract $\gamma \approx 490 \mathrm{eV} \AA^{3}$. This value agrees closely with theoretical calculations yielding $\gamma$ in $n$-InSb/InAlSb quantum wells as a function of well thickness, which have predicted $\gamma$ $\approx 425 \mathrm{eV} \AA^{3}$ for a 25 -nm-wide well. ${ }^{28}$ The value $\gamma$ $=490 \mathrm{eV} \AA^{3}$ signifies, in fact, an upper bound for $\gamma$ at $0.4 \mathrm{~K}$, resulting from the assumption of dominant remote-ionized impurity scattering. Accounting for other scattering mechanisms which yield a smaller ratio $\left(\tau_{1} / \tau_{3}\right)$, such as phonon scattering or interface roughness scattering, ${ }^{30}$ would result in a somewhat smaller $\gamma$. We stress however that at $T=0.4 \mathrm{~K}$

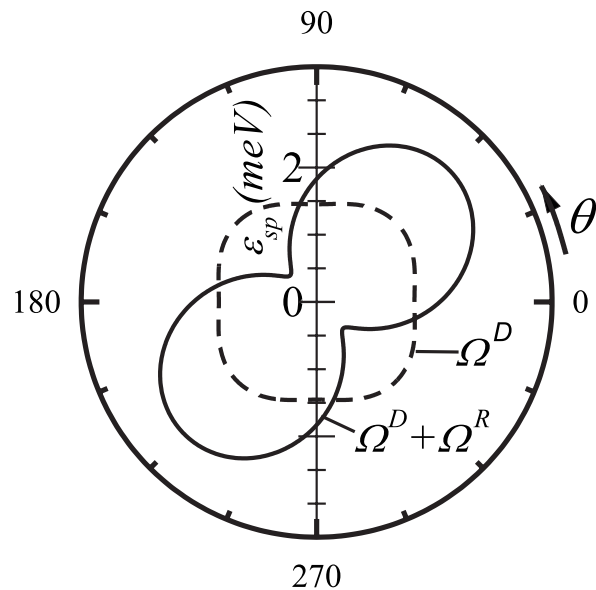

FIG. 6. Calculated SOI-induced energy splitting $\boldsymbol{\epsilon}_{s p}=2 \hbar|\boldsymbol{\Omega}|$ of the conduction band at $k_{F}\left(k_{F}^{2}=2 \pi N_{s}\right)$ as a function of momentum direction. The plot is obtained by substituting $\gamma=490 \mathrm{eV} \AA^{3}, \alpha$ $=0.03 \mathrm{eV} \AA$, and $\left\langle k_{z}^{2}\right\rangle=8 \times 10^{15} \mathrm{~m}^{-2}$ into Eqs. (1a)-(1c).

remote-ionized impurity scattering, rather than phonon or interface roughness scattering, likely determines $\left(\tau_{1} / \tau_{3}\right)$ and we hence accept $y \approx 490 \mathrm{eV} \AA^{3}$.

From the extracted values for $\alpha \approx 0.03 \mathrm{eV} \AA$ and $\gamma$ $=490 \mathrm{eV} \AA^{3}$ at $0.4 \mathrm{~K}$ we calculate the average spin-orbitinduced energy splitting of the conduction band at $k_{F}$ as $\left\langle\epsilon_{s p}\right\rangle \approx 1.8 \mathrm{meV}$, and an average spin-precession frequency of $2\langle|\boldsymbol{\Omega}|\rangle=2.7 \times 10^{12} \mathrm{~s}^{-1}$. The dependence of $\boldsymbol{\epsilon}_{s p}$ on momentum direction, depicted in Fig. 6, reveals an anisotropy of $\epsilon_{s p}$ in the plane of the 2DES. $\epsilon_{s p}$ ranges between $\approx 0.6$ and $\approx 2.8 \mathrm{meV}$ depending on the direction of $\boldsymbol{k}$. The angular dependence results from the $3 \theta$ dependence of $\boldsymbol{\Omega}_{3 \theta}^{D}$ as compared to the $\theta$ dependence of $\boldsymbol{\Omega}^{R}$. The fact that both terms have substantial values in the 2DES produces a marked anisotropy.

Beyond $T=0.4 \mathrm{~K}, 2\left|\boldsymbol{\Omega}^{R}\right|^{2} \tau_{1} \sim N_{s} \tau_{p}$ is found to have a negligible $T$ dependence reflecting the fact that, as observed in Hall measurements, $N_{s}$ and $\tau_{p}$ remain almost constant $\left(<2 \%\right.$ change) over this range of $T$. In contrast, $2\left|\boldsymbol{\Omega}_{3 \theta}^{D}\right|^{2} \tau_{3}$ $\sim N_{s}^{3} \tau_{3}$ increases by a factor $\approx 1.7$ from 0.4 to $20 \mathrm{~K}$. In order to explain the dependence on $T$ of spin relaxation in III-V semiconductors, Ref. 30 has shown that $2\left|\Omega^{R}\right|^{2} \tau_{1}$ and $2\left|\boldsymbol{\Omega}_{3 \theta}^{D}\right|^{2} \tau_{3}$ can be multiplied by $T$-dependent factors, to become

$$
\begin{gathered}
\left|\boldsymbol{\Omega}^{R}\right|^{2} \tau_{1} \rightarrow\left|\boldsymbol{\Omega}^{R}\right|^{2} \tau_{p}\left(1-e^{-E_{F} \beta}\right)^{-1}, \\
\left|\boldsymbol{\Omega}_{3 \theta}^{D}\right|^{2} \tau_{3} \rightarrow \frac{\left|\boldsymbol{\Omega}_{3 \theta}^{D}\right|^{2}}{\left(E_{F} \beta\right)^{2}} \frac{\tau_{p}\left(\tau_{3} / \tau_{1}\right)}{1-e^{-E_{F} \beta}} \frac{J_{\nu+3}\left(\beta \mu_{0}\right)}{J_{\nu+1}\left(\beta \mu_{0}\right)},
\end{gathered}
$$

where $\beta=1 / k_{B} T$ (with $k_{B}$ the Boltzmann constant), the chemical potential $\mu_{0}=\ln \left(e^{E_{F} \beta}-1\right) / \beta, 4 J_{n}(z)=\int_{0}^{\infty} x^{n} \operatorname{sech}^{2}[(x$ $-z) / 2] d x$, and where $\nu$ depends on the operational scattering mechanism. For weakly screened charged impurity scattering considered here, $\nu=2 .{ }^{30}$ Using values for $|\alpha|$ and $\gamma$ obtained at $0.4 \mathrm{~K}$ we find that Eq. (6a) accurately describes $2\left|\boldsymbol{\Omega}^{R}\right|^{2} \tau_{1}$ over the experimental range of $T$. However, Eq. (6b) predicts a weaker $T$ dependence of $2\left|\boldsymbol{\Omega}_{3 \theta}^{D}\right|^{2} \tau_{3}$ than experimentally observed (Fig. 4). A portion of the additional $T$ dependence 
results from changes in $\left(\tau_{3} / \tau_{1}\right)$ from increased phonon scattering with $T$. We suggest that electron-electron $(e-e)$ scattering may also contribute to the observed $T$ dependence of $2\left|\boldsymbol{\Omega}_{3 \theta}^{D}\right|^{2} \tau_{3}$. Although $e$-e scattering has little effect on $\tau_{p}$, recently a large impact of $e$ - $e$ scattering on spin relaxation has been suggested in high- $\mu \mathrm{GaAs} / \mathrm{AlGaAs}$ quantum wells. ${ }^{49,50}$ As compared to doped semiconductor thin films, remotely doped heterostructures experience a much reduced scattering rate from ionized impurities, and hence effects arising from $e-e$ scattering ${ }^{49,50}$ are more evident in high- $\mu$ 2DESs. Equation (3) does not explicitly include the contribution from $e-e$ scattering to spin relaxation, yet its effect can be approximated as follows. Since the parameter $b_{s}$ expresses the spinrelaxation rate, we expect the effect of $e$ - $e$ scattering on spin relaxation to affect $b_{s}$. Any contribution to $b_{s}$ not included in $b_{s}^{\prime}$ is grouped into the term $2\left|\Omega_{3 \theta}^{D}\right|^{2} \tau_{3}$, suggesting that the spin-relaxation rate from $e$-e scattering $\left(1 / \tau_{s}^{e e}\right)$ should be added to $b_{s}$, namely, $b_{s} \rightarrow b_{s}^{*}=b_{s}+\hbar /\left(4 e D H \tau_{s}^{e e}\right)$, similar to the inclusion of spin relaxation via the Elliot-Yafet mechanism. ${ }^{10}$ Experimentally observed then is the value of $2\left|\boldsymbol{\Omega}_{3 \theta}^{D}\right|^{2} \tau_{3}^{*}$ derived from $b_{s}^{*}$, with $2\left|\boldsymbol{\Omega}_{3 \theta}^{D}\right|^{2} \tau_{3}^{*}=2\left|\boldsymbol{\Omega}_{3 \theta}^{D}\right|^{2} \tau_{3}$ $+\left(1 / \tau_{s}^{e e}\right)$. As $T \rightarrow 0, e-e$ scattering is expected to vanish. Thus $1 / \tau_{s}^{e e}$ is not expected to alter the values of $|\alpha|$ and $\gamma$ obtained from the $0.4 \mathrm{~K}$ values of $2\left|\boldsymbol{\Omega}^{R}\right|^{2} \tau_{1}$ and $2\left|\boldsymbol{\Omega}_{3 \theta}^{D}\right|^{2} \tau_{3}$. Yet, our observation suggests that the $T$ dependence of $2\left|\boldsymbol{\Omega}_{3 \theta}^{D}\right|^{2} \tau_{3}^{*}$ may be a consequence of an increase in $1 / \tau_{s}^{e e}$ with $T$, not negligible except at the lowest $T$.

In summary, we have extracted the SOI terms active in an $n$-InSb/InAlSb 2DES, by fitting magnetotransport measurements with AL theory. Accurate modeling of the measured $\Delta \sigma(H)-\Delta \sigma(0)$, requires the presence of both the Rashba SOI $\boldsymbol{\Omega}^{R}$ and the Dresselhaus $\boldsymbol{\Omega}_{3 \theta}^{D}$ contributions, and requires an estimate of the predominant momentum scattering mechanism. It is found that in the heterostructure the Rashba coefficient $\alpha \approx 0.03 \mathrm{eV} \AA$ and the Dresselhaus coefficient $\gamma$ $\approx 490 \mathrm{eV} \AA^{3}$.

\section{ACKNOWLEDGMENTS}

This work is supported by DOE through Award No. DEFG02-08ER46532 and by NSF through Awards No. DMR0618235 and No. DMR-0520550. *kallaher@vt.edu

theremans@vt.edu

${ }^{1}$ T. Ashley, L. Buckle, S. Datta, M. T. Emeny, D. G. Hayes, K. P. Hilton, R. Jefferies, T. Martin, T. J. Phillips, D. J. Wallis, P. J. Wilding, and R. Chau, Electron. Lett. 43, 777 (2007).

${ }^{2}$ J. M. S. Orr, P. D. Buckle, M. Fearn, C. J. Storey, L. Buckle, and T. Ashley, New J. Phys. 9, 261 (2007).

${ }^{3}$ S. A. Solin, T. Thio, D. R. Hines, and J. J. Heremans, Science 289, 1530 (2000).

${ }^{4}$ S. A. Solin, D. R. Hines, A. C. H. Rowe, J. S. Tsai, Yu. A. Pashkin, S. J. Chung, N. Goel, and M. B. Santos, Appl. Phys. Lett. 80, 4012 (2002).

${ }^{5}$ H. Chen, J. J. Heremans, J. A. Peters, A. O. Govorov, N. Goel, S. J. Chung, and M. B. Santos, Appl. Phys. Lett. 86, 032113 (2005).

${ }^{6}$ B. L. Al'tshuler, A. G. Aronov, A. I. Larkin, and D. E. Khmel'nitskii, Zh. Eksp. Teor. Fiz. 81, 768 (1981) [Sov. Phys. JETP 54, 411 (1981)].

${ }^{7}$ G. Bergmann, Phys. Rep. 107, 1 (1984).

${ }^{8}$ S. Hikami, A. I. Larkin, and Y. Nagaoka, Prog. Theor. Phys. 63, 707 (1980).

${ }^{9}$ A. Zduniak, M. I. Dyakonov, and W. Knap, Phys. Rev. B 56, 1996 (1997).

${ }^{10}$ W. Knap, C. Skierbiszewski, A. Zduniak, E. Litwin-Staszewska, D. Bertho, F. Kobbi, J. L. Robert, G. E. Pikus, F. G. Pikus, S. V. Iordanskii, V. Mosser, K. Zekentes, and Yu. B. Lyanda-Geller, Phys. Rev. B 53, 3912 (1996).

${ }^{11}$ J. B. Miller, D. M. Zumbühl, C. M. Marcus, Y. B. Lyanda-Geller, D. Goldhaber-Gordon, K. Campman, and A. C. Gossard, Phys. Rev. Lett. 90, 076807 (2003).

${ }^{12}$ S. McPhail, C. E. Yasin, A. R. Hamilton, M. Y. Simmons, E. H. Linfield, M. Pepper, and D. A. Ritchie, Phys. Rev. B 70, 245311 (2004).

${ }^{13}$ S. A. Studenikin, P. T. Coleridge, N. Ahmed, P. J. Poole, and A.
Sachrajda, Phys. Rev. B 68, 035317 (2003).

${ }^{14}$ D. D. Bykanov, A. M. Kreshchuk, S. V. Novikov, T. A. Polyanskaya, and I. G. Savel'ev, Semiconductors 32, 985 (1998).

${ }^{15}$ T. Koga, J. Nitta, T. Akazaki, and H. Takayanagi, Phys. Rev. Lett. 89, 046801 (2002).

${ }^{16}$ V. A. Guzenko, M. Akabori, Th. Schäpers, S. Cabañas, T. Sato, T. Suzuki, and S. Yamada, Phys. Status Solidi C 3, 4227 (2006).

${ }^{17}$ P. D. Dresselhaus, C. M. A. Papavassiliou, R. G. Wheeler, and R. N. Sacks, Phys. Rev. Lett. 68, 106 (1992).

${ }^{18}$ G. L. Chen, J. Han, T. T. Huang, S. Datta, and D. B. Janes, Phys. Rev. B 47, 4084 (1993).

${ }^{19}$ G. M. Minkov, A. V. Germanenko, O. E. Rut, A. A. Sherstobitov, L. E. Golub, B. N. Zvonkov, and M. Willander, Phys. Rev. B 70, 155323 (2004).

${ }^{20}$ T. Hassenkam, S. Pedersen, K. Baklanov, A. Kristensen, C. B. Sorensen, P. E. Lindelof, F. G. Pikus, and G. E. Pikus, Phys. Rev. B 55, 9298 (1997).

${ }^{21}$ S. Pedersen, C. B. Sørensen, A. Kristensen, P. E. Lindelof, L. E. Golub, and N. S. Averkiev, Phys. Rev. B 60, 4880 (1999).

${ }^{22}$ R. L. Kallaher and J. J. Heremans, Phys. Rev. B 79, 075322 (2009).

${ }^{23}$ A. M. Kreshchuk, S. V. Novikov, T. A. Polyanskaya, and I. G. Savel'ev, Semiconductors 31, 391 (1997).

${ }^{24}$ M. Oszwaldowski, T. Berus, and V. K. Dugaev, Phys. Rev. B 65 , 235418 (2002)

${ }^{25}$ L. E. Golub, Phys. Rev. B 71, 235310 (2005).

${ }^{26} \mathrm{~S}$. V. Iordanskii, Yu. B. Lyanda-Geller, and G. E. Pikus, Pis'ma Zh. Eksp. Teor. Fiz. 60, 199 (1994) [JETP Lett. 60, 206 (1994)]

${ }^{27}$ F. G. Pikus and G. E. Pikus, Phys. Rev. B 51, 16928 (1995).

${ }^{28}$ A. M. Gilbertson, M. Fearn, J. H. Jefferson, B. N. Murdin, P. D. Buckle, and L. F. Cohen, Phys. Rev. B 77, 165335 (2008).

${ }^{29}$ J. Kainz, U. Rössler, and R. Winkler, Phys. Rev. B 68, 075322 (2003).

${ }^{30}$ J. Kainz, U. Rössler, and R. Winkler, Phys. Rev. B 70, 195322 
(2004).

${ }^{31}$ N. S. Averkiev, L. E. Golub, and M. Willander, J. Phys.: Condens. Matter 14, R271 (2002).

${ }^{32}$ N. S. Averkiev, L. E. Golub, and M. Willander, Semiconductors 36, 91 (2002).

${ }^{33}$ P. Pfeffer and W. Zawadzki, Phys. Rev. B 68, 035315 (2003).

${ }^{34}$ G. Dresselhaus, Phys. Rev. 100, 580 (1955).

${ }^{35} \mathrm{R}$. Winkler, Spin-Orbit Coupling Effects in Two-Dimensional Electron and Hole Systems (Springer-Verlag, Berlin, 2003).

${ }^{36}$ M. Cardona, N. E. Christensen, and G. Fasol, Phys. Rev. B 38, 1806 (1988).

${ }^{37}$ G. Lommer, F. Malcher, and U. Rössler, Phys. Rev. Lett. 60, 728 (1988).

${ }^{38}$ G. A. Khodaparast, R. E. Doezema, S. J. Chung, K. J. Goldammer, and M. B. Santos, Phys. Rev. B 70, 155322 (2004).

${ }^{39}$ A. R. Dedigama, D. Jayathilaka, S. H. Gunawardana, S. Q. Murphy, M. Edirisooriya, N. Goel, T. D. Mishima, and M. B. Santos, in Narrow Gap Semiconductors 2007: Proceedings of the 13th International Conference, edited by B. N. Murdin and S. K. Clowes, Springer Proceedings in Physics Vol. 119, p. 35 (Springer, Netherlands, 2008).

${ }^{40}$ J. H. Davies, The Physics of Low-Dimensional Semiconductors: An Introduction (Cambridge University Press, Cambridge,
1998).

${ }^{41}$ N. Dai, F. Brown, R. E. Doezema, S. J. Chung, K. J. Goldammer, and M. B. Santos, Appl. Phys. Lett. 73, 3132 (1998).

${ }^{42}$ N. Dai, G. A. Khodaparast, F. Brown, R. E. Doezema, S. J. Chung, and M. B. Santos, Appl. Phys. Lett. 76, 3905 (2000).

${ }^{43}$ K. S. Romanov and N. S. Averkiev, Zh. Eksp. Teor. Fiz. 128, 811 (2005) [Sov. Phys. JETP 101, 699 (2005)].

${ }^{44}$ W. H. Lau, J. T. Olesberg, and M. E. Flatté, Phys. Rev. B 64, 161301(R) (2001).

${ }^{45}$ S. J. Chung, M. A. Ball, S. C. Lindstrom, M. B. Johnson, and M. B. Santos, J. Vac. Sci. Technol. B 18, 1583 (2000).

${ }^{46}$ S. Washburn and R. A. Webb, Adv. Phys. 35, 375 (1986).

${ }^{47}$ F. Nihey, S. W. Hwang, and K. Nakamura, Phys. Rev. B 51, 4649 (1995).

${ }^{48}$ J. M. S. Orr, A. M. Gilbertson, M. Fearn, O. W. Croad, C. J. Storey, L. Buckle, M. T. Emeny, P. D. Buckle, and T. Ashley, Phys. Rev. B 77, 165334 (2008).

${ }^{49}$ M. A. Brand, A. Malinowski, O. Z. Karimov, P. A. Marsden, R. T. Harley, A. J. Shields, D. Sanvitto, D. A. Ritchie, and M. Y. Simmons, Phys. Rev. Lett. 89, 236601 (2002).

${ }^{50}$ W. J. H. Leyland, G. H. John, R. T. Harley, M. M. Glazov, E. L. Ivchenko, D. A. Ritchie, I. Farrer, A. J. Shields, and M. Henini, Phys. Rev. B 75, 165309 (2007). 